https://doi.org/10.1007/s00350-021-5835-9

\section{Anmerkung zu LSG Nordrhein-Westfalen, Beschl. v. 17.7.2020 - L 11 KA 23/19 B ER (SG Düsseldorf) \\ Eugen Ewig}

Der Beschluss ist sorgfältig begründet und steckt den rechtlichen Rahmen für das noch anhängige Hauptsacheverfahren ab. Er gibt aber auch Anlass, für die Plausibilitätsprüfungen im Rahmen der sachlich-rechnerischen Prüfung gemäß $\$ 106 d$ SGB V verfahrens- und materiell-rechtliche Fragen zu stellen. Das gilt zum einen für die Dauer des Verfahrens. Während die Anordnung der aufschiebenden Wirkung durch das SG Düsseldorf noch innerhalb von eineinhalb Monaten erfolgte, lagen zwischen der Beschwerde der Antragsgegnerin v. 4.3.2019 und dem Beschluss v. 17. 7. 2020 gut 15 Monate. Bei einer solchen Verfahrensdauer kann man nicht mehr von einem angemessenen einstweiligen Rechtsschutz sprechen, vor allem, wenn man berücksichtigt, dass eine Honorarkürzung in Höhe von mehr als 190.000,00 EUR drohte.

Das gilt zum anderen für die Durchführung der Plausibilitätsprüfung. Die Antragstellerin wurde erstmalig in der üblichen Anhörung des Prüfgremiums damit konfrontiert, dass die Leistungen nach EBM 01773, 01774 und 01775 als vorgeburtliche Risikoabklärung nach $\$ 3$ Nr. 3 GenDG eingestuft wurden und damit die Kürzung aller abgerechneten Ziffern wegen einer fehlenden schriftlichen Einwilligung gem. $\$ 8$ Abs. 1 GenDG im Prüfungszeitraum im Raum stand. Soweit ersichtlich gibt es bundesweit keine vergleichbaren Fälle, in denen eine Einwilligung der Patientinnen nach dem Gendiagnostikgesetz als notwendiger Bestandteil der Leistungen nach den genannten GOPen angesehen wird. Für die Pränatalmediziner, die dies überwiegend bisher auch nicht so gesehen haben dürften, drohen, falls sich die Rechtsauffassung der KÄV durchsetzt, Honorarkürzungen in Millionenhöhe. Im Sinne einer rechtzeitigen Aufklärung und eines fairen Verfahrens läge es, wenn die KÄVen, sofern sie Leistungslegenden entgegen ihrer bisher üblichen Praxis grundsätzlich neu auslegen, ihre Mitglieder zwingend frühzeitig zu informieren, bevor diese durch nachträgliche Honorarkürzungen beschwert werden.

Erfrischend eindeutig stellt das LSG materiellrechtlich klar, dass Dokumentationspflichten, die Ärzten außerhalb der vertragsärztlichen Bestimmungen auferlegt werden, nicht im Wege der Auslegung in den EBM hineininterpretiert werden dürfen. Eine sachlich-rechnerische Prüfung hat sich ihrem Sinn und Zweck nach auf die Prüfung der Einhaltung der Leistungslegenden $\mathrm{zu}$ beschränken. Das LSG lässt daher auch erhebliche Zweifel durchblicken, die gegensätzliche Auffassung des 1. Senats des BSG, demzufolge bei einer fehlerhaften Einwilligung eines Patienten zur Behandlung der Vergütungsanspruch des Krankenhauses entfallen soll, auch im Vertragsarztrecht anzuwenden. Das ist schon deshalb zutreffend, weil im Unterschied zur Abrechnung des Krankenhauses mit der Krankenkasse eine Prüfung der Abrechnung des Vertragsarztes im Wege des Honorarbescheides erfolgt. Würde man der Rechtsauffassung der KÄV konsequent folgen, hieße das, dass Honorarkürzungen im Rahmen von sachlich-rechnerischen Prüfungen auch auf unwirksame Aufklärungen bei operativen Eingriffen gestützt werden könnten.

Schutzzweck der nach $\$ 8$ Abs. 1 GenDG im Fall einer vorgeburtlichen Risikoabklärung geforderten Aufklärung und schriftlichen Einwilligung ist die Wahrung des

Rechtsanwalt Eugen Th. Ewig, Fachanwalt für Medizinrecht, EWIG NORD + PARTNER mbB Rechtsanwälte, Büchelstr. 50, 53227 Bonn, Deutschland
Selbstbestimmungsrechts des Patienten. Allein dieser darf darüber entscheiden, ob eine genetische Untersuchung durchgeführt werden soll und ob er Kenntnis von dem Ergebnis dieser Untersuchung erlangen will oder nicht. Unter dem Begriff „,genetische Untersuchung“ werden Methoden zusammengefasst, die unmittelbaren Aufschluss über die genetische Ausstattung eines Organismus oder Organs geben können. Die Untersuchungen dienen der sicheren Feststellung menschlicher genetischer Eigenschaften mittels genetischer Analyse ( $\$ 3 \mathrm{Nr}$. 1a GenDG) oder einer vorgeburtlichen Risikoabklärung ( $\$ 3 \mathrm{Nr}$. 1b GenDG). Eine genetische Untersuchung ist nach dem Wortlaut des $\$ 3$ Abs. 1 GenDG durch den Untersuchungszweck, also auf die Gewinnung genetischer Erkenntnisse, gekennzeichnet.

Untersuchungen nach den GOPen 01773, 01774 und 01775 EBM sind daher nach wohl herrschender Auffassung nicht als genetische Untersuchung zu klassifizieren, weil sie nicht gezielt auf die Erforschung genetischer Ursachen ausgerichtet sind (Schillhorn, Gendiagnostikgesetz, Kommentar für die Praxis, 2. Aufl. 2017, \&3, Rdnr. 10). Aus gynäkologischer Sicht gibt es eine Vielzahl von Ursachen und Verdachtsmomenten, die eine weiterführende sonographische Diagnostik nach den Indikationen der Anlagen 1c und 1d Mutterschafts-Richtlinien rechtfertigen und nicht mit genetischen Ursachen zusammenhängen, wie z.B. Alkohol- oder Nikotinabusus, Gewalteinwirkung, unklare Strukturen im Screening etc. Zu berücksichtigen ist hierbei, dass werdende Mütter leicht zu verunsichern sind und eine regelmäßige zwingende Aufklärung nach dem GenDG im Rahmen der weiterführenden sonografischen Diagnostik vielfach zusätzliche Ängste und Sorgen um das ungeborene Kind auslösen dürfte.

Die durchgängige Einordnung der Leistungen nach den GOP 01773, 01774 und 01775 EBM als genetische Untersuchung wirft außerdem datenschutzrechtliche Probleme auf. Denn nach $\$ 11$ Abs. 1 GenDG sind die Erkenntnisse aus einer genetischen Untersuchung streng geschützt. Sie dürfen nur der betroffenen Personen und nur durch den untersuchenden Arzt mitgeteilt werden. Nach $\$ 11$ Abs. 1 GenDG wäre es dem Vertragsarzt daher untersagt, Patientenakten mit entsprechenden Inhalten der KÄV im Rahmen einer Plausibilitätsprüfung zur Verfügung zu stellen. \106d SGB V ist keine geeignete Rechtsgrundlage, um Ausnahmen hiervon zu rechtfertigen.

\section{Einführung des ab dem 1.1.2019 gültigen} $\S 301$ Abs. 2 S. 4 SGB V (heute §301 Abs. 2 S. 6 SGB V) durch das Pflegepersonal-Stärkungsgesetz

\section{v. 11.12.2018}

SGB V §§109 Abs. 4 S. 3, 301 Abs. 2 v. 11.12. 2018 (heute $\S 301$ Abs. 2 S. 6 SGB V); GG Art. 20 Abs. 2, Abs. 3, 87 Abs. 3, 100 Abs. 1 S. 1; KHG $\S 17$ b Abs. 1, Abs. 2; PpSG, Nr. 8-98b OPS 2014

Dem BVerfG werden folgende Fragen zur Entscheidung vorgelegt:

1. Verstößt der durch das Pflegepersonal-Stärkungsgesetz v. 11.12.2018 (BGB1. I S. 2394) hinzugefügte $\$ 301$ Abs. 2 S. 4 SGB V, gültig ab dem 1.1.2019, insoweit gegen den Gesetzesvorbehalt der Art. 20 Abs. 2 und 3, 87 Abs. 3 GG, als die in $\$ 301$ Abs. 2 S. 4 SGB V

Eingesandt und bearbeitet von

Justiziar Dr. iur. Stephan-Georg Zacharias,

IKK Südwest,

Trierer Straße 4, 66111 Saarbrücken, Deutschland 